ORIGINAL

\title{
CARACTERÍSTICAS Y ANÁLISIS DE LA SUPERVIVENCIA DE LAS PERSONAS ATENDIDAS EN EL PROGRAMA DE ATENCIÓN DOMICILIARIA DEL ÁREA BÁSICA DE SALUD RAVAL NORD, BARCELONA, 1993-2002
}

Josep María Segura Noguera, Núria Bastida Bastús, Núria Martí Guadaño, Montserrat Riba Romeva, Mireira Marsá Cantero y Santiago Lancho Lancho

Centro de Atención Primaria Dr. Lluís Sayé. Equipo de Atención Primaria Raval Nord, Ciutat Vella. Barcelona

\section{RESUMEN}

Fundamentos: El aumento de la esperanza de vida ha conducido a un progresivo envejecimiento de la población y a una creciente atención sóciosanitaria a domicilio. El objetivo del presente estudio es describir la población de enfermos crónicos domiciliarios atendiequipo de atención primaria de un Centro de Salud urbano, estudiar la frecuentación y analizar la supervivencia de las personas atendidas en el mismo.

Métodos: Estudio descriptivo. Los sujetos de estudio fueron los 1.357 enfermos crónicos incluidos desde el inicio de un programa de atención domiciliaria (mayo 94-diciembre 2002). En primer lugar se realizó un análisis descriptivo de las características principales de esta población. Posteriormente, mediante el método de los riesgos proporcionales de Cox, se valoraron los factores asociados con la supervivencia, y asimismo se elaboraron las correspondientes curvas de probabilidad de supervivencia acumulada Kaplan-Meier.

Resultados: Predominio de población femenina $(68 \%)$, y una media de edad de 82 años (DE: 11 años). Destacan la falta de ascensores $(76 \%)$, y la soledad (18\%). Presentan dependencia absoluta para todas las actividades de la vida diaria un $11 \%$ de los enfermos, mientras que aproximadamente un $20 \%$ muestra severas alteraciones cognitivas. En relación con la atención domiciliaria, se superó el objetivo del programa de 2-3 visitas/enfermo/año para el personal médico y de 4-6 para el personal de enfermería. La vacunación antigripal se mantuvo siempre por encima del objetivo del $70 \%$, mientras que la vacunación antitetánica aumentó anualmente.

Conclusiones: La población atendida en este programa se caracteriza por ser muy envejecida, con una alta mortalidad y que requiere atención domiciliaria continuada. Durante los 10 años estudiados se han logrado la mayor parte de los objetivos prefijados.

Palabras clave: Servicios de atención de salud a domicilio. Atención primaria de salud. Analisis de supervivencia.

Correspondencia:

Josep $\mathrm{M}^{\mathrm{a}}$ Segura Noguera

C/ Villarroel, $149.4^{\circ} 1^{\mathrm{a}}$

08036 Barcelona

\section{ABSTRACT}

Characteristics and Analysis of the Survival of Individuals Cared for under the Homecare Program in the Raval Nord Health Area in Barcelona, Spain.1993-2002

Background: Longer life expectancy has led to a progressive aging of the population and to growing sociosanitary homecare. This study is aimed at describing the population of patients suffering from chronic diseases having been provided with homecare throughout the first ten years that homecare was available by a primary care team at an urban Healthcare Center, to study how often this type of care was used and to analyze the survival of those individuals having been provided with this care.

Methods: A descriptive study. The subjects under study were 1,357 chronically-ill patients included from the very start of a homecare program (May 1994-December 2002). Firstly, a descriptive analysis was made the main characteristics of this population. Afterward, using Cox's proportional hazards model, the factors linked to survival were assessed, as well as having plotted the corresponding cumulative survival probability curves using the Kaplan-Meier method.

Results: Predominant female population (68\%), and an average age of 82 (DE: 11 years). Worth of special note were the lack of elevators $(76 \%)$ and loneliness $(18 \%)$. Eleven totally dependent for all of whilst approximately $20 \%$ revealed severe cognitive disorders. With regard to the homecare, the program target of 2-3 visits/patient/year for the medical staff and off 4-6 visits/patient/year for the nursing staff were surpassed. The flu vaccine injections were always above the $70 \%$ target, whilst the tetanus vaccine injections increased yearly.

Conclusions: The population cared for under this program is characterized by being greatly aged, with a high death rate and requiring continuous homecare. During the ten years studied, most of the objectives previously set were achieved.

Keywords: Primary health care. Home care services. Survival analisys. 


\section{INTRODUCCIÓN}

El aumento de la esperanza de vida experimentado en los últimos años (determinado por mejoras en las condiciones sanitarias, en el diagnóstico y tratamiento de las enfermedades, y por el desarrollo económicosocial), ha conducido a un progresivo envejecimiento de la población ${ }^{1,2}$. Gran parte de esta población presenta enfermedades crónicas o invalidantes que han determinado una creciente atención sociosanitaria a domicilio $^{3-5}$, fenómeno que implica que se destinen más recursos, tanto humanos como materiales, para atender esta demanda. Esta realidad conlleva la necesidad de dar respuestas eficientes desde la atención primaria a los principales problemas de salud de la población. Así, la atención domiciliaria es una función propia del equipo de atención primaria (EAP) desde el mismo origen de la reforma, con una importancia creciente ${ }^{6,7}$. Aunque en determinadas ocasiones pueda ser necesaria la ayuda de otros profesionales de soporte, la asistencia a domicilio se realiza prácticamente en su totalidad desde la atención primaria. Una circunstancia biopsicosocial, como el hecho de que el enfermo no puede salir de su domicilio, se constituye en la razón de ser de este tipo de asistencia.

Diversos estudios han mostrado un aumento de población senil dependiente secundario al progresivo envejecimiento. Según datos de la Enquesta de Salut de Barcelona del año $1992^{8}$, el 20\% de las personas mayores de 65 años presentaba algún tipo de limitación en las actividades de la vida diaria, cifra que según la Enquesta de Salut de Barcelona del año $2000^{9}$ se ha incrementado hasta valores superiores al $30 \%$.

El programa de atención domiciliaria (programa ATDOM) es el instrumento necesario para que el EAP pueda realizar una atención a domicilio de forma correcta y organiza$\mathrm{da}^{10,11}$. Para ello es necesario realizar una planificación previa teniendo en cuenta los recursos disponibles ${ }^{12-14}$, y realizar una eva- luación periódica según los objetivos definidos previamente. A partir de la elaboración del Programa Marc d'Atenció Domiciliaria del Servei Català de la Salut ${ }^{15-17}$ se ha incrementado de forma importante el número de EAP que utilizan un programa ATDOM en su actividad habitual, tal como ha señalado una encuesta reciente ${ }^{18}$. Una etapa fundamental en todo programa ATDOM es la evaluación periódica de los objetivos que han sido previamente definidos por el $\mathrm{EAP}^{19}$. Uno de los objetivos principales de nuestro programa, cuya cobertura ha oscilado entre el 5 y el $8 \%$ de la población senil, es realizar una descripción integral y detallada tanto de las características de los enfermos crónicos domiciliarios como de la atención domiciliaria prestada por el equipo. Además, con la sospecha que un mayor deterioro, tanto físico como mental, podría relacionarse con una menor supervivencia, se decidió analizar estos aspectos.

El objetivo del presente estudio es describir las características de los enfermos crónicos atendidos en el programa de atención domiciliaria y la frecuentación, así como analizar en esta población la supervivencia que hace referencia a los 10 primeros años (1993-2002) de funcionamiento de un EAP.

\section{SUJETOS Y MÉTODO}

El centro de asistencia primaria (CAP) «Dr. Lluís Sayé», ubicado en «Ciutat Vella», inició su actividad el 14 de diciembre del año 1992. Actualmente, el EAP está integrado por 11 unidades básicas asistenciales (UBA) de medicina general y 2 de pediatría, repartidas en turnos de mañana y tarde. También dispone de médico odontólogo, asistente social y personal administrativo. La población atendida es la que reside en el barrio de «Raval Nord». Cada UBA, que consta de un médico y un diplomado en enfermería, presta asistencia en el centro 4 días a la semana, dedicando el quinto día a la atención domiciliaria, tanto espontánea como programada,

Rev Esp Salud Pública 2003, Vol. 77, N. ${ }^{\circ} 5$ 
que desde mayo de 1994 se realiza mediante un programa ATDOM. Además, el personal de enfermería dedica otro día de la semana a tiempo parcial para esta actividad.

El barrio de «Raval Nord» tiene una población total censada de 17.501 habitantes según datos del año 2000, de los que 4.446 personas son iguales o mayores de 65 años $(25,4 \%)$. Otra característica del barrio es el importante incremento de la población inmigrante en los últimos años (superior al 30\%). Este hecho ha comportado que la tasa de población igual o mayor de 65 años haya disminuido un $3 \%$ en la última década $(28,4 \%$ el año 1991, 29,6\% el año 1996, 25,4\% el año 2000). Además, otro dato ya conocido antes de realizar el estudio, es que esta zona se caracteriza por un déficit importante de ascensores en los edificios.

Durante estos 10 años se ha incluido en el programa ATDOM a todas las personas que requerían atención en el propio domicilio, debido a presentar una dificultad en la capacidad de desplazamiento que les impedía acudir hasta el CAP. Los datos de cada enfermo son registrados en la correspondiente ficha de control y evaluación, la cual es archivada centralizadamente por el equipo y clasificada por cada UBA. La ficha presenta dos partes bien diferenciadas, una corresponde a la descripción sociosanitaria del individuo, siendo cumplimentada cuando una persona es incluida en el programa, mientras que en la otra parte se anotan todas las visitas realizadas en el domicilio del enfermo por cualquier profesional del EAP (médico, enfermería, asistente social). Cuando el enfermo es dado de alta en el programa se codifica la causa, y la ficha es guardada en una parte del fichero correspondiente a pasivo. Para la evaluación se utilizan los datos registrado en esta ficha, y la selección de indicadores para la evaluación se determina según los objetivos anuales del programa.

Se han incluido en el estudio a todos los enfermos crónicos atendidos en su domicilio e incluidos en el registro desde el inicio del programa ATDOM, en concreto desde mayo de 1994 a diciembre de 2002 (104 meses). Los únicos criterios de exclusión fueron los de vivir fuera del barrio de «Raval Nord», personas que solicitaban visitas a domicilio de complacencia no justificadas (ya que podían desplazarse perfectamente hasta el CAP), o personas que se negaran a ser visitadas a domicilio. Aunque la visita habitual trata de un seguimiento de los problemas de salud del enfermo, en la primera visita (generalmente conjunta entre médico y personal de enfermería) se realiza también una valoración del estado físico y mental del enfermo. Además, el personal de enfermería realiza, si procede, el plan de las curas a realizar. La valoración de la soledad ha sido un tema prioritario para la asistente social del centro, que anualmente realiza una nueva valoración de la situación de los enfermos crónicos domiciliarios con cada UBA. En ocasiones, se ha dispuesto de la ayuda del Equipo de Soporte en Atención Domiciliaria (PADES) formado por un médico, personal de enfermería y asistente social, para la atención de los enfermos clasificados como terminales en el grupo de enfermos incluidos.

Mediante el registro ya descrito se han analizado diversas variables referentes tanto a las características de los enfermos (edad, sexo, estado civil, origen de la demanda, categoría de inclusión en el programa, patología predominante, grado de autonomía, estado mental, soledad-compañía, condiciones de vivienda, y tiempo de inclusión en el programa) como a las visitas realizadas (motivo, duración, tipo de visita y profesional que acude al domicilio). Cuando el enfermo es dado de alta en el programa se describe la causa de la misma (mejoría que permite desplazarse hasta el CAP, defunción, cambio de domicilio o ingreso en residencias fuera del barrio, y otras causas). Asimismo, se calculó la tasa anual de frecuentación de visitas domiciliarias según cada profesional. Para evaluar la frecuentación anual de visitas se utilizó el indicador de intensi- 
dad de ATDOM, definido como el número de visitas dividido por el número de meses que los enfermos permanecen en el programa cada año, multiplicado por doce. La valoración de la capacidad física fue realizada mediante el índice de Katz ${ }^{20-22}$, escala que clasifica el grado de autonomía desde independiente para todas las funciones (A) hasta dependiente para todas ellas (G), a partir de seis actividades básicas de la vida diaria (baño, vestirse, uso WC, movilidad, continencia esfinteriana y alimentación). En la categoría $\mathrm{H}$ del índice de Katz se incluyen casos no clasificables según las restantes categorías, y en la práctica se comporta como una categoría con una dependencia leve. La valoración de la capacidad mental fue realizada mediante el Short Portable Mental Status Questionnarie de Pfeiffer, test muy útil por su rapidez de aplicación junto a una aceptable sensibilidad y especifidad ${ }^{23-26}$, y que sirve para clasificar a los enfermos según su estado cognitivo (intacto; deterioro leve, moderado, severo), aunque en ocasiones el deterioro severo no permite realizar el test.

Los datos del registro fueron introducidos en una base de datos (Dbase III Plus) y posteriormente tratados con el paquete estadístico SPSS 10.0 para Windows, tanto para la estadística descriptiva, como para analizar la supervivencia. Por lo que respecta al análisis de supervivencia, se han agrupado los valores de las variables más complejas, como el estado cognitivo y el índice de Katz. Asimismo, también se han agrupado algunas de la categorías iniciales de las variables relativas a cada grupo (patología crónica, atdom transitoria, demencias, enfermos terminales, y grupos de riesgo para atdom), y a la variable soledad-compañía (vive solo, vive acompañado con personas mayores o menores de 60 años). En la variable soledad-compañía se han unificado en una sola categoría los que viven acompañados. Para el estudio del estado cognitivo se han fusionado los deterioros leves con los moderados, y los impracticables se han reconsiderado en la categoría de severos. En el índice de Katz, se han simpli- ficado las categorías en independiente (índice Katz A), dependiente parcial (índices de Katz B, C, D, E, F, H), y dependiente total (índice de Katz $\mathrm{G}$ ). El método de los riegos proporcionales de Cox se utilizó para determinar el riesgo relativo (RR) estimado de mortalidad según las diferentes categorías recodificadas de las variables estudiadas, mientras que las correspondientes curvas de probabilidad de supervivencia acumulada se calcularon según el método de KaplanMeier. En todo momento el nivel de significación utilizado fue $\mathrm{p}=0,05$. Finalmente, se realizó un análisis ajustado con todas las variables asociadas a la supervivencia, para valorar si todas ellas conservaban su significación.

\section{RESULTADOS}

Desde el comienzo del programa ATDOM en mayo de 1994 hasta el mes de diciembre de 2002 han sido atendidos 1.357 enfermos crónicos en su domicilio. Un $18 \%$ de los enfermos estaba ingresado en residencias del mismo barrio, mientras que otro $18 \%$ vivía solo. Aunque el $64 \%$ restante vivía acompañado en su piso, un $33 \%$ lo hacía con personas mayores de 60 años. Como se muestra en la tabla 1, existe un predominio del sexo femenino que se da sobre todo a partir de los 75 años $(68,3 \%)$ (p < 0,001). También predomina el estado civil de viudo/a $(50,7 \%)$, seguido del de casado/a $(32,1 \%)$, solteros $(14,8 \%)$ y otros $(2,4 \%)$, existiendo para este variable diferencias significativas según el sexo $(\mathrm{p}<0,001)$. Entre los casados predominan los varones $(61,0 \%)$, mientras que entre los solteros las mujeres $(69,4 \%)$. Respecto al estado de viudedad existe un predominio de las mujeres $(87,1 \%)$. La edad media es de 82 años (DE: 11), presentando diferencias estadísticamente significativas ( $\mathrm{p}<0,001)$ según el sexo (mujeres 84 ; varones 77).

La distribución según el grupo de inclusión en el programa es: patología crónica 
Tabla 1

Enfermos crónicos domiciliarios según edad y sexo

\begin{tabular}{|c|c|c|c|c|}
\hline \multirow{2}{*}{$\begin{array}{c}\text { Grupos de edad } \\
<35 \text { años }\end{array}$} & \multicolumn{2}{|c|}{ Viarones } & \multicolumn{2}{|c|}{ Mlujeres } \\
\hline & 6 & $54,6 \%$ & 5 & $45.4 \%$ \\
\hline 35-64 aโ̂os & 57 & $69.5 \%$ & 25 & $30,5 \%$ \\
\hline 65-74 ab̆os & 102 & $53.4 \%$ & 89 & $46.6 \%$ \\
\hline $75-84$ aกิธง & 146 & $292 \%$ & 353 & $70,8 \%$ \\
\hline - 85 años & 119 & $20,7 \%$ & 455 & $79.3 \%$ \\
\hline Total & 430 & $31.7 \%$ & 927 & $683 \%$ \\
\hline
\end{tabular}

$(78,4 \%)$, enfermos terminales $(7,9 \%)$, demencias $(6,3 \%)$, atdom transitoria $(5,7 \%)$, y usuarios en grupos de riesgo $(1,7 \%)$. El elevado número de enfermos incluidos en el programa ha presentado una tendencia ligeramente decreciente, excepto en el último año (tabla 2). El promedio de meses que los enfermos han estado incluidos en el programa ha presentado una ligera tendencia a aumentar progresivamente, permaneciendo alrededor de los 8,5 meses. Referente a las causas de las $1.091(80,4 \%)$ altas en el programa 194 (14,3\%) enfermos ingresaron en residencias y centros sociosanitarios fuera de nuestra zona, $130(9,6 \%)$ se trasladaron de domicilio, $116(8,5 \%)$ recuperaron su autonomía, mientras que $570(42,0 \%)$ fallecieron. En los $81(6,0 \%)$ casos restantes se perdió el contacto con el enfermo y, por tanto, se desconoce la causa de alta del programa.

La demanda de ATDOM fue realizada en la mayoría de casos por la familia $(61 \%)$ o el mismo EAP (26\%), mientras que del $13 \%$ restante en la mitad de los casos fueron los servicios sociales los que informaron. El motivo de visita más frecuente fue el seguimiento del enfermo (60\%). En un $75 \%$ de las visitas la duración fue de 15-30 minutos. El $31,7 \%$ de los enfermos eran hipertensos y el porcentaje de diabéticos era de $17,8 \%$. Las patologías más predominantes fueron los problemas cardiovasculares $(24,0 \%)$, osteoarticulares $(17,1 \%)$, neurológicos $(16,1 \%)$, endocrinos $(12,7 \%)$, respiratorios $(7,2 \%)$, neoplásicos $(7,1 \%)$, digestivos $(5,2 \%)$, urológicos $(4,7 \%)$, psiquiátricos $(3,4 \%)$, y otros $(2,5 \%)$. Un $27,4 \%$ de los enfermos tenían algún tipo de incontinencia esfinteriana mientras que menos de un 5\% de los enfermos presentaron úlceras por decúbito. El $76 \%$ de los edificios donde viven estos enfermos no disponían de ascensor. El promedio de habitaciones y personas en los domicilios fueron 3 y 2,2 respectivamente. A destacar que en un $25 \%$ de los domicilios existían animales domésticos, y un $38 \%$ no disponía de baño completo. Los cuidadores predominantes eran mujeres de mediana edad (hijas), seguidos por remunerados, y mujeres de avanzada edad (esposas). La distribución de los cuidadores identificados era: hijos/hijas (31\%), remunerados $(24 \%)$, cónyuge $(18 \%)$, padres $(3 \%)$, vecinos $(4 \%)$, otros familiares $(12 \%)$ y otros $(8 \%)$.

En la figura 1 se puede observar como se lograron cada año los objetivos del programa (indicador de intensidad de ATDOM de 2-3 visitas/año para el personal médico y de 4-6 visitas/año para el personal de enfermería, sin contar los enfermos que vivían en las residencias del barrio), e incluso las visitas, tanto las de enfermería como las médicas, superaron claramente este objetivo en los 


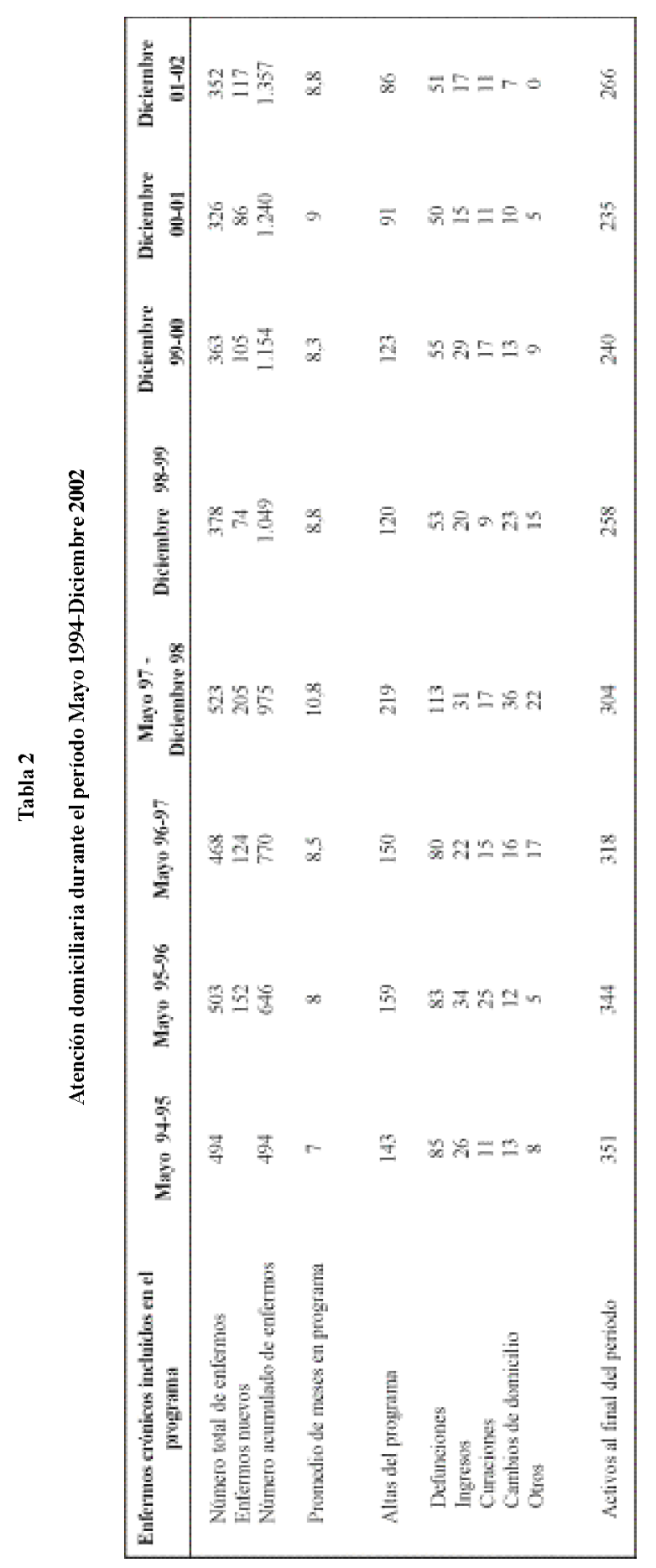


Figura 1

Visitas anuales por enfermo

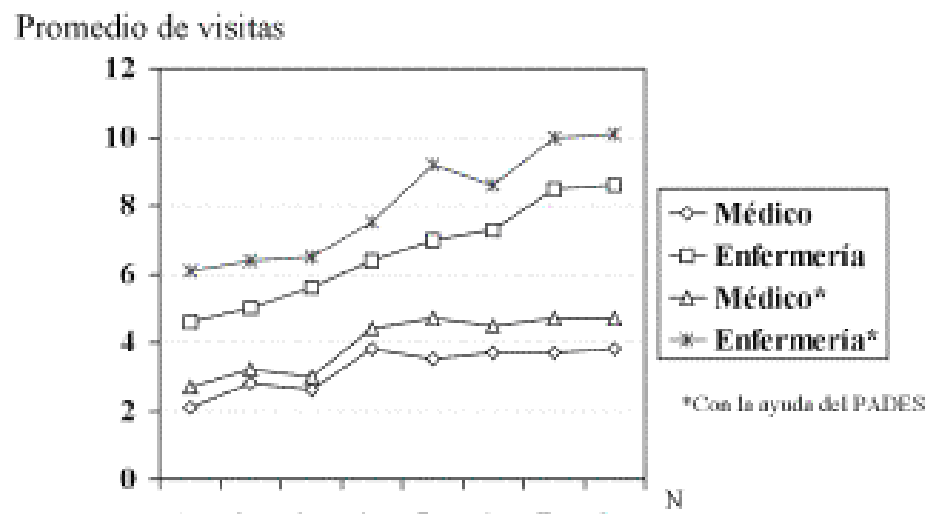

1a. 2a. 3a. 4a. 5a. 6a. 7a. 8a.

Evaluación

últimos años, especialmente si se tiene en cuenta la ayuda del equipo PADES.

Un $10,9 \%$ de los enfermos eran dependientes para todas las actividades básicas de la vida diaria (índice de $\mathrm{Katz} « \mathrm{G} »$ ), un $58,9 \%$ presentaron dependencia de forma parcial (índices de $\mathrm{Katz} « \mathrm{~B} » 22,6 \%, 《 \mathrm{C} »$ $13,1 \%, \ll \mathrm{D} » 6,6 \%,\langle\mathrm{E} » 4,1 \%,\langle\mathrm{~F} » 7,8 \%$, $\ll \mathrm{H} » 4,7 \%)$, mientras que un $30,2 \%$ eran independientes en todas las actividades (índice de Katz «A»). Un 54,1\% de los enfermos no presentaron alteraciones en el test mental utilizado, mientras que tenían deterioro leve un $16,4 \%$, moderado un $9,7 \%$ y severo un $19,8 \%$ de los enfermos (en un $4,1 \%$ casi impracticable). Como era de esperar, es significativo $(\mathrm{p}<0,001)$ el mejor grado de autonomía y estado cognitivo de los enfermos que viven solos.

Los factores que mostraron una relación significativa $(p<0,001)$ con una peor supervivencia fueron el sexo masculino (RR:1,67), la necesidad de estar acompañado (RR: 2,39), el estado cognitivo deteriorado (leve-moderado, RR: 1,74; severo, RR: 2,71 ), un peor grado de autonomía (dependencia parcial, RR: 1,63; dependencia total,
RR: 3,54), y el grupo de tipo de enfermo terminal (en su mayoría por patología neoplásica, RR: 7,72). En la tabla 3 se muestra el análisis de los riesgos proporcionales de Cox para los diferentes valores de las variables estudiadas. En las figuras 2 a 6 se muestran las curvas de probabilidad de supervivencia acumulada de Kaplan y Meier según los factores pronósticos mencionados. Se encontraron asimismo diferencias significativas $(p<0,001)$ en la edad media según la supervivencia de los enfermos (sí, 73 años; no, 79 años). Los 405 enfermos sin seguimiento completo no presentaron diferencias significativas en las variables estudiadas. El análisis ajustado por las variables asociadas a la supervivencia, no experimentó cambios de interés respecto al análisis crudo (todas las variables mantuvieron su significación, excepto el estado cognitivo).

Finalmente, es importante remarcar que la vacunación antigripal ha sido siempre igual o superior al objetivo del $70 \%$, mientras que el porcentaje de enfermos visitados en más de 3 ocasiones a los que se ha administrado la vacuna antitetánica ha aumentado progresivamente (desde un $15 \%$ a un $65 \%)$. 
Josep Ma Segura Noguera et al

Tabla 3

Riesgos Relativos estimados de mortalidad de los enfermos crónicos domiciliarios según las variables estudiadas

\begin{tabular}{|c|c|c|}
\hline Variables & IC $95 \%$ & $\mathbf{P}$ \\
\hline \multicolumn{3}{|l|}{ Sexo } \\
\hline Mujer & entegoria de referencia & - \\
\hline Varơn & $1,67 \quad 1,41-1,97$ & $<0,001$ \\
\hline \multicolumn{3}{|l|}{ Girupo } \\
\hline Pstologia crónica & categoria de referencia & - \\
\hline Atdom transitoria & $0,16 \quad 0,09-0,29$ & $<0,001$ \\
\hline Demencias & $0,80-1,47$ & 0,002 \\
\hline Enfermo terminal & $6,12 \cdot 9,74$ & $<0,001$ \\
\hline \multicolumn{3}{|l|}{ Soledad-compañia } \\
\hline Vive solo & estegoria de referencia & - \\
\hline Vive con companis & $2,39 \quad 1,80-3,18$ & $<0,001$ \\
\hline \multicolumn{3}{|l|}{ Estado Cognitivo } \\
\hline Intacis & categoris de referencia & - \\
\hline Deterioro leve-moderads & $1,74 \quad 1,13-2.69$ & 0,012 \\
\hline Deterioro severo & $1,75 \cdot 4,19$ & $<0,001$ \\
\hline \multicolumn{3}{|l|}{ Indice de Katz } \\
\hline Indeperxdiente & cstegoria de referencia & $\cdot$ \\
\hline Dependiente parcial & $1.63 \quad 1.29-2.05$ & $<0,(001$ \\
\hline Dependiente total & $2,63 \cdot 4,76$ & $<0,001$ \\
\hline
\end{tabular}

Figura 2

Supervivencia según sexo

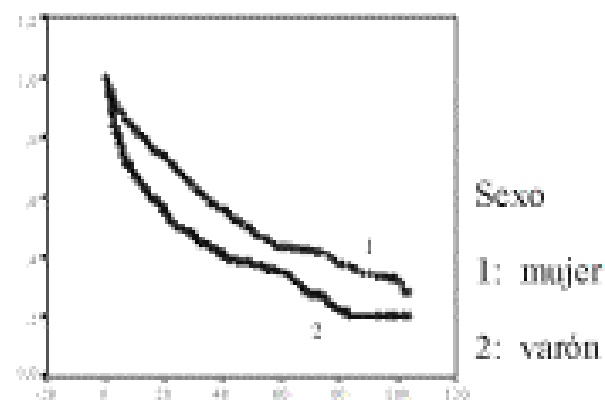

Tiempo de seguimiento en meses 
CARACTERÍSTICAS Y ANÁLISIS DE LA SUPERVIVENCIA DE LAS PERSONAS ATENDIDAS EN EL PROGRAMA DE...

Figura 3

Supervivencia según soledad-compañía

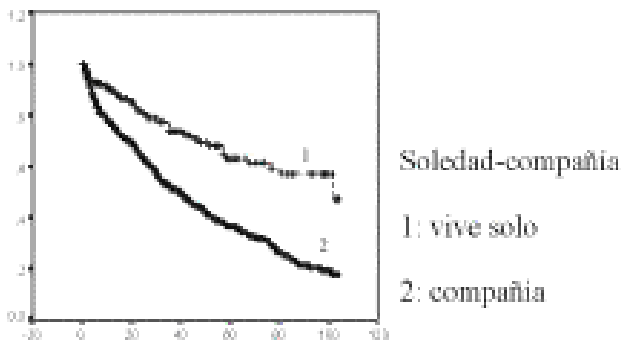

Tiempo de seguimiento

en meses

Figura 4

Supervivencia según grupo

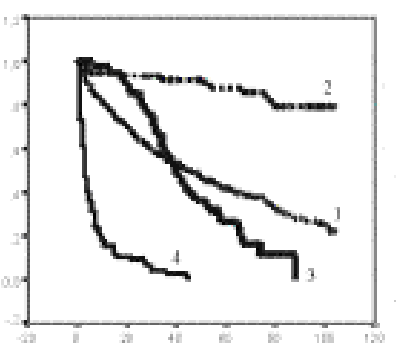

Grupo diana

1: patologia cróniça

2: atdom transitoria

3; demencias

4: terminales

Tiempo de seguimiento

en meses

Figura 5

Supervivencia según estado cognitivo

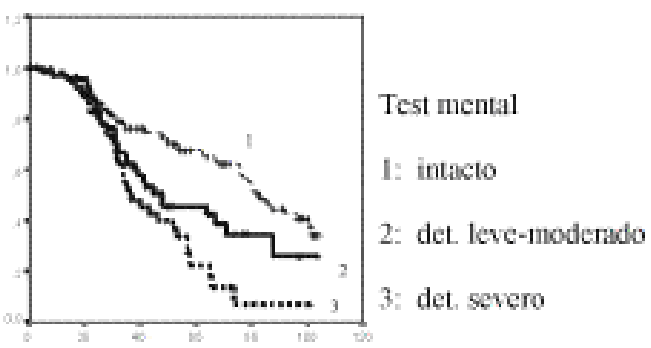

Tiempo de seguimiento en neses 


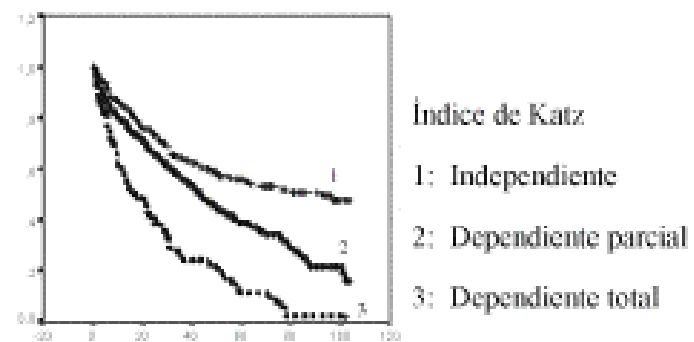

Tiempo de seguimiento en meses

\section{DISCUSIÓN}

La importancia creciente de la atención domiciliaria dentro de la atención primaria de salud es un fenómeno que en el futuro condicionará que se destinen cada vez más recursos tanto humanos como materiales. Este hecho es especialmente relevante en áreas como la nuestra con un envejecimiento muy importante, y por tanto con una importante demanda de atención domiciliaria. Las condiciones de una gran parte de las viviendas del barrio, con importantes barreras arquitectónicas como la falta de ascensores (76\%), junto con la soledad en la que vive parte de la población anciana (18\%) con un grado de autonomía limitado, condicionan la gran demanda de atención domiciliaria existente. Por estas razones se ha desarrollado durante este período un programa de ayudas técnicas que permite la cesión de diferentes materiales (sillas, caminadores, muletas, elevadores wc, camas articuladas...), y así poder ayudar a mejorar el grado de autonomía de nuestra población senil dependiente.

El programa ATDOM ha demostrado ser una herramienta eficiente ${ }^{27-30}$ que facilita el seguimiento del enfermo y permite un adecuado registro tanto de la evolución del enfermo como de las actividades que se realizan en el domicilio (visitas, curas, vacunas.
Los principales indicadores que se han ido monitorizando hacen referencia a la periodicidad de visitas por parte de los profesionales, y a las vacunaciones antigripal y antitetánica. Es destacable que durante estos 10 años también se han desarrollado por parte de profesionales de nuestro centro los proyectos «Cuidem els cuidadors informals» $\mathrm{y}$ «Riscos de la llar», y el ya mencionado programa de ayudas técnicas. Respecto a la cuestión de si la implantación del programa ATDOM es adecuada, es importante mencionar que está muy condicionada en nuestro caso por la falta de ascensores. Por ello, aunque un $30 \%$ de los enfermos eran independientes para todas las actividades básicas de la vida diaria, no podían salir de sus domicilios para desplazarse hasta el CAP.

El perfil básico de los enfermos incluidos en el programa ATDOM muestra un importante predominio de mujeres viudas, hecho concordante con la mayoría de estudios rea$\operatorname{lizados}^{31-34}$ debido a la mayor esperanza de vida de la mujer. El porcentaje de enfermos con buena autonomía es inferior al de los trabajos que estudian el grado de autonomía en la población senil ${ }^{22,33,35}$. En cambio, si comparamos este dato con algún estudio ${ }^{34}$ que lo analiza exclusivamente en la población de crónicos domiciliarios es superior, dato atribuible a que en muchos casos la imposibilidad de desplazarse al CAP es debida a la fal- 
ta de ascensores y a la pluripatología que presentan los enfermos.

Nosotros utilizamos la medición del grado de autonomía según el índice de Katz por haber sido demostrada su utilidad en diversos estudios ${ }^{22,24,36}$. En un estudio previo ${ }^{37}$, este índice demostró ser un buen predictor tanto de la supervivencia de los enfermos como de la frecuentación de visitas. Así, un peor grado de autonomía condicionó una mayor frecuentación de visitas tanto por el personal médico como por el personal de enfermería. Para este último colectivo también destacaron la presencia de úlceras por decúbito y la diabetes mellitus como factores asociados a la frecuentación. La soledad fue el factor más asociado a las visitas de la asistente social.

Asimismo, otro estudio realizado en nuestro ámbito ${ }^{38}$, referente al cuidador, describió que es significativo el porcentaje de malestar psíquico detectado en los cuidadores, como ocurre en otros estudios de similares características $^{39-41}$. Mediante las escalas de ansiedad y depresión de Goldberg (EADG) ${ }^{42,43}$, se detectó malestar psíquico prácticamente en la mitad de los cuidadores encuestados ( $82 \mathrm{de}$ $167)$, con alteraciones en ambas escalas (ansiedad y depresión) en un $27 \%$, en la escala de ansiedad en un $18 \%$ y en la escala de depresión en un $4 \%$. El porcentaje de enfermos crónicos atendidos en su domicilio con un severo deterioro mental es ligeramente inferior al hallado en otros artículos ${ }^{32,45}$. En nuestro caso el hallazgo tanto de enfermos con deterioro cognitivo importante como de cuidadores con alteraciones en el test de Goldberg, conllevó el estudio de los prime$\operatorname{ros}^{45}$ y la introducción en los cursos de formación de cuidadores de los segundos. Otros aspectos que asimismo han sido de nuestro interés con anterioridad fueron las caídas ${ }^{45} \mathrm{y}$ el consumo de psicofármacos ${ }^{46}$ en los enfermos crónicos atendidos por el programa.

Respecto a los resultados en el apartado de supervivencia, es importante resaltar que son concordantes con otros estudios en lo que se refiere a la menor supervivencia de los varones ${ }^{44,47}$. Uno de estos estudios ${ }^{47}$ también destaca la importancia de la valoración del grado de autonomía, además de resaltar la importancia de la opinión inicial del médico en relación a la posible supervivencia, variable que nosotros no hemos estudiado. Algunos hechos anecdóticos de nuestro estudio tienen su explicación. Así, la mejor supervivencia de los enfermos que viven solos viene explicada por su asociación a un mejor grado de autonomía y estado cognitivo. Los enfermos a los que no se pudo realizar la valoración del SPMSQ (por tratarse de demencias muy evolucionadas) se han considerado equivalentes a demencia severa. Por otra parte, es evidente la diferente evolución esperable en cuanto a mortalidad de los distintos grupos de inclusión. Aunque estas diferencias se deben a las características de los enfermos incluidos en el programa, creemos importante su descripción. Finalmente, es importante resaltar que la mayoría de factores asociados a la supervivencia de estos enfermos ya se evidenciaban en el análisis realizado a los dos años de seguimiento ${ }^{37}$, aunque con el tiempo se han suavizado ligeramente las diferencias existentes según el índice de Katz.

Debemos aclarar que todo el trabajo realizado en «Raval Nord» en atención domiciliaria durante estos 10 años se hizo previamente al inicio del programa «Salut a Casa» ${ }^{48}$ implementado recientemente en la ciudad de Barcelona, y que por tanto integra también el programa ATDOM de «Raval Nord». Este hecho ha requerido de la consiguiente adaptación de nuestro programa a los objetivos, indicadores, y escalas de valoración consensuadas para toda la ciudad de Barcelona. El seguimiento incompleto de un $29 \%$ de los enfermos, por cambios de domicilio, ingreso en residencias fuera de nuestro ámbito, y otras causas desconocidas, no supone una limitación de los resultados, ya que el análisis de estos enfermos no difería en las variables predictoras de superviven- 
cia. Por otra parte, es importante subrayar que la tasa de frecuentación se refiere siempre a visitas domiciliarias; así se evita el sesgo producido por la existencia de visitas esporádicas (no registradas en la base de datos) realizadas en el mismo CAP cuando un enfermo con buena autonomía puede ser acompañado. Por último, creemos importante resaltar que aunque son pocos los estudios en el ámbito de la atención primaria que estudian la supervivencia de los enfermos, el programa ATDOM permite un adecuado registro para su análisis. Así, una adecuada valoración integral de la atención domiciliaria permite detectar problemas que precisan una redefinición de nuevos objetivos.

\section{AGRADECIMIENTOS}

Agradecemos el trabajo realizado por todos los miembros del EAP «Raval Nord» en la implantación del programa ATDOM, lo que ha permitido la realización de este estudio. Asimismo, también agradecemos a los enfermos crónicos y a sus familias su buena predisposición a colaborar en el mismo.

\section{BIBLIOGRAFÍA}

1. Gillick MR. Long-term care options for the frail elderly. J Am Geriatr Soc 1989; 37: 1198-203.

2. Ministerio de Asuntos Sociales. La tercera edad en España: aspectos cuantitativos. Proyecciones de la población española de 60 y más años para el período 1986- 2010. Madrid: Ministerio de Asuntos Sociales; 1991.

3. Taylor RB. Home Care. En: Taylor RB. Family Medicine: principles and practicle. New York: Springer Verlag Inc; 1978.

4. Luker KA, Perkins ES. The elderly at home: service needs and provision. Journal of the Royal College of General Practitioner 1987; 37:248-50.

5. Freer CB. Screening the elderly. BMJ 1990; 300: 1447-8.

6. López Pisa RM, Maymó N, Giró E. Atención domiciliaria. En: Martín Zurro A, Cano Pérez JF.
Manual de Atención Primaria. Barcelona: Doyma (2. ${ }^{\mathrm{a}}$ ed.); 1989.

7. Torra JE. Atención domiciliària y atención primaria de salut. Rev Rol Enferm 1989; 125:37-43.

8. Institut Municipal de la Salut. Enquesta de Salut de Barcelona 1992-1993. Barcelona: Institut Municipal de la Salut; 1994.

9. Institut Municipal de la Salut. Enquesta de Salut de Barcelona 2000. Barcelona: Institut Municipal de la Salut; 2000.

10. Borrell F. Programas de salud en atención primaria. Necesidad de innovaciones metodológicas. Aten Primaria 1986; 3(2):91-6.

11. López Pisa RM, Maymó Pijuan N. Programación de la atención domiciliaria. Monografías clínicas de Aten Primaria 1991; 9(3):21-23.

12. Pinault R, Daveuly C. La planificación sanitària. Conceptos, métodos, estrategias. Barcelona: Masson (2 $2^{\mathrm{a}}$ ed); 1989.

13. Segura JM, Bastida N, Martí N, González C. Atención domiciliaria: estudio previo a la implantación de un programa. Aten Primaria 1994; 14(6):852.

14. Segura JM, Sanz F, Bastida N, Martí N, González C, Guerrero M. Descripción y validación de un modelo predictivo de la distribución geográfica de los enfermos crónicos domiciliarios. Aten Primaria 1996; 17(4): 292-4.

15. Programa Marc d'Atenció a Domicili ATDOM. Divisió d'Atenció Primària i Hospitalària de l'Àrea Sanitària del Servei Català de la Salut. Barcelona: Servei Català de la Salut; 1994.

16. Forés M, Lacasa C, Ris H. El programa d'atenció domiciliària en un EAP. Elements del programa marc. Salut Catalunya 1992; 6(3): 95-9.

17. Lacasa C, Forés M, Ris H. Desenvolupament de l'atenció domiciliària a Catalunya. Enquesta als EAP. Salut Catalunya 1993; 7(5): 222-5.

18. Grupo ATDOM de la Sociedad Catalana de Medicina Familiar y Comunitaria. Aproximación a la atención domiciliaria. ¿Cómo trabaja la sanidad pública catalana la atención domiciliaria? Aten Primaria $2003 ; 31: 473-9$.

19. Salleras L. La investigación epidemiológica en la evaluación de los programas de salud. Aten Primaria $1988 ; 5$ : 122-34.

20. Katz S, Ford AB, Moskowitz RW, Jackson BA, Jaffe MW. Studies of illnes in the aged: the índex of

Rev Esp Salud Pública 2003, Vol. 77, N. ${ }^{\circ} 5$ 
ADL, a standarized measure of biological and psychosocial function. JAMA 1963; 85:914-919.

21. Katz S, Downs TD, Cash $\mathrm{H}$ et al. Progress in the development of the índex of ADL. Gerontologist 1970; 10:20-30.

22. Alvarez M, Alaiz A, Brun E, Cabañeros J, Calzón M, Cosío I et al. Capacidad funcional de enfermos mayores de 65 años, según el índice de Katz. Fiabilidad del método. Aten Primaria 1992; 10: 812-6.

23. Vinyoles E, Recasens JM, Salvador B, Sáez A. La demencia: una visión desde la atención primaria. Aten Primaria 1992; 10(5): 789-93.

24. González JI, Salgado A. Manejo del enfermo anciano en atención primaria. Línes guía. Aten Primaria 1992; 9(4): 219-26.

25. Kane RA, Kane RL. Evaluación de las necesidades en los ancianos. Guía práctica sobre los instrumentos de medición. Barcelona: SG Editores; 1993.

26. González Montalvo JI, Rodríguez Mañas L, Ruipérez Cantera I. Validación del cuestionario de Pfeiffer y la escala de incapacidad mental de la Cruz Roja en la detección del deterioro mental en los enfermos externos en un servicio de Geriatría. Rev Esp Geriatr Gerontol 1992; 27: 129-33.

27. Contel JC, González M. Reflexión sobre la implementación de programas de atención domiciliaria. Enfermería Clínica 1995; 5(5): 205-11.

28. Lindoso T, Valencia S, Fernández A, Álvarez S, Álvarez JA. Monitorizar un programa de atención domiciliaria. Rev Rol Enferm 1994; 188: 13-16.

29. Espinosa JM, Muñoz F. Evaluación de los programas de atención domiciliaria en atención primaria de salud. Cuadernos de Gestión 1995; 1(1): 24-32.

30. López Pisa RM, Agüera Villar B. Atenció domiciliària: avaluació de 5 anys de funcionament del programa. Salut Catalunya 1995; 9(5): 203-207.

31. Bilbao I, Gastaminza AM, García JA, Quindimil JA, López JL, Huidobro L. Los enfermos domiciliarios crónicos y su entorno en atención primaria de salud. Aten Primaria 1994; 13(4): 188-190.

32. Sanz C, Morente M, Calvo C, Gallego P, Carra B. Valoración psico-física del anciano. Rev Rol Enferm 1995; 206: 17-20.

33. Monte C, Alonso JC, Cabal A, Iglesias A. Capacidad funcional en mayores de 80 años. Aten Primaria $1995 ; 15(3)$ : 176-178.

34. Hernando J, Iturioz P, Torán P, Arratibel I, Arregui A, Merino M. Problemas y necesidades de salud de

Rev Esp Salud Pública 2003, Vol. 77, N. ${ }^{\circ} 5$ los enfermos crónicos domiciliarios. Aten Primaria 1992; 9(3): 133-136.

35. Benítez MA, Hernández P, Barreto J. Evaluación geriátrica exhaustiva en atención primaria: valoración de su utilidad. Aten Primaria 1994; 13(1): 2026.

36. Cruz Jentoft AJ. El Índice de Katz. Rev Esp Geriatr Gerontol 1991; 26: 338-348.

37. Segura JM, Bastida N, Martí N, Riba M. Enfermos crónicos domiciliarios. Análisis descriptivo y de supervivencia a los 2 años de la implantación de un programa de atención domiciliaria. Aten Primaria 1997; 19(7): 351-356.

38. Segura JM, Bastida N, Martí N, Riba M. Los enfermos crónicos domiciliarios y su repercusión en los cuidadores principales. Aten Primaria 1998; 21: 431-436.

39. Puig C, Hernández LM, Gervás JJ. Los enfermos crónicos recluidos en el domicilio y su repercusión en la familia: una investigación cualitativa. Aten Primaria 1992; 10(1): 550-552.

40. Rubio Montañés ML, Sánchez Ibáñez A, Ibáñez Estella JA, Galve Royo F, Martí Talens N, Mariscal Bernal J. Perfil medicosocial del cuidador crucial. ¿Se trata de un enfermo oculto? Aten Primaria 1995; 16(4): 181-186.

41. Seira MP, Aller A, Calvo A. Morbilidad sentida y diagnosticada en cuidadores de enfermos inmovilizados de una zona de salud rural. Rev Esp Salud Pública 2002; 76: 713-721.

42. Goldberg D, Bridges K, Duncan-Jones P et al. Detecting anxiety and depression in general medical settings. Br Med J 1988; 97: 897-899.

43. Montón C, Pérez MJ, Campos R, García J, Lobo A, GMPPZ. Escalas de ansiedad y depresión de Goldberg: una guía de entrevista eficaz para la detección de malestar psíquico. Aten Primaria 1993; 12(6): 345-349.

44. González Montalvo JI, Jaramillo Gómez E, Rodríguez Mañas L, Guillén Llera F, Salgado Alba A. Estudio evolutivo de los enfermos en asistencia geriátrica domiciliaria a los 18 meses. Rev Clín Esp 1990; 187: 165-169.

45. Segura JM, Barreiro MC, Bastida N, Collado D, González M, Linares C, et al. Enfermos crónicos domiciliarios: valoración integral físico-cognitiva y caídas durante tres años de seguimiento. Aten Primaria 2000; 25: 297-301.

46. Segura JM, Barreiro MC, Bastida N, Collado D, 
González M, Linares C, et al. Enfermos crónicos domiciliarios y consumo de psicofármacos. Aten Primaria 2000; 26: 620-623.
47. Palomo L, Gervas J. Mortalidad a los 2 años en enfermos crónicos confinados en el domicilio. Aten Primaria 2000; 25: 176-180. 